\title{
Associations of consumption of fruits and vegetables during pregnancy with infant birth weight or small for gestational age births: a systematic review of the literature
}

\author{
This article was published in the following Dove Press journal: \\ International Journal of Women's Health \\ 20 October 2014 \\ Number of times this article has been viewed
}

\author{
Mary M Murphy' \\ Nicolas Stettler ${ }^{1,2}$ \\ Kimberly M Smith' \\ Richard Reiss ${ }^{3}$ \\ 'Exponent, Inc., Center for \\ Chemical Regulation and Food \\ Safety, Washington, DC, USA; ${ }^{2}$ The \\ Lewin Group, Falls Church,VA, \\ USA; ${ }^{3}$ Exponent, Inc., Center for \\ Chemical Regulation and Food \\ Safety, Alexandria, VA, USA
}

Correspondence: Mary M Murphy Exponent, Inc., Center for Chemical Regulation and Food Safety, I I 50 Connecticut Avenue NW, Suite I1 100, Washington, DC 20036, USA Tel + I 2027724953

Fax + I 2027724979

Email mmurphy@exponent.com

\begin{abstract}
Maternal nutrition is recognized as one of the determinants of fetal growth. Consumption of fruits and vegetables is promoted as part of a healthful diet; however, intakes are typically lower than recommended levels. The purpose of this study was to systematically review results from studies examining the relationship between maternal consumption of fruits and vegetables during pregnancy with infant birth weight or risk for delivering a small for gestational age baby. A comprehensive search of PubMed and EMBASE was conducted and abstracts were screened using predefined criteria. Eleven relevant studies were identified and systematically reviewed, including six prospective cohort studies, three retrospective cohort studies, and two case-control studies. Seven studies were conducted in cohorts from highly developed countries. One prospective study from a highly developed area reported increased risk for small for gestational age birth by women with low vegetable intakes (odds ratio 3.1; $95 \%$ confidence interval $1.4-6.9 ; P=0.01$ ); another large prospective study reported a $10.4 \mathrm{~g}$ increase in birth weight per quintile increase in fruit intake ( $95 \%$ confidence interval 6.9-3.9; $P<0.0001$ ) and increases of 8.4 or $7.7 \mathrm{~g}$ per quintile intake of fruits and vegetables (combined) or fruits, vegetables, and juice (combined), respectively. One retrospective study reported an association between low fruit intake and birth weight. In less developed countries, increased vegetable or fruit intake was associated with increased birth weight in two prospective studies. Overall, limited inconclusive evidence of a protective effect of increased consumption of vegetables and risk for small for gestational age birth, and increased consumption of fruits and vegetables and increased birth weight among women from highly developed countries was identified. Among women in less developed countries, limited inconclusive evidence suggests that increased consumption of vegetables or fruits may be associated with higher infant birth weight. The available evidence supports maternal consumption of a variety of fruits and vegetables as part of a balanced diet throughout pregnancy.
\end{abstract}

Keywords: birth weight, small for gestational age, pregnancy, fruit, vegetable

\section{Introduction}

While fetal growth is largely determined by non-nutritional factors, such as genetics, placental function, maternal age, stature, parity, immunologic response to the pregnancy, blood pressure, infections, chronic diseases, prenatal care, smoking, and altitude, optimal maternal nutrition also appears to play a role. ${ }^{1,2}$ The importance of folic acid and iron intake in optimal pregnancy outcomes is well established based on intervention studies, and some experimental data suggest that other micro- or macronutrients may be important, especially among undernourished women., 
Findings from observational studies suggest that overall diet quality during pregnancy, or patterns of dietary intake consistent with a nutrient-dense diet, are positively associated with birth outcomes, including increased birth weight and reduced risk for infants delivered small for gestational age (SGA). ${ }^{2,5-8}$ Increased maternal consumption of specific nutrient-dense foods such as milk and fish has also been positively associated with birth weight outcomes..$^{9-12}$

Fruits and vegetables are nutrient-dense foods and key sources of a number of essential nutrients, including potassium, magnesium, dietary fiber, folate, and vitamins $A$ and $C$; fruits and vegetables also contain a variety of other bioactive substances that may play a role in health. ${ }^{13}$ Observational studies of fruit and vegetable intake during pregnancy and birth weight or the incidence of SGA births have not consistently reported a significant association. ${ }^{14-16}$

Consumption of fruits and vegetables is promoted as part of a nutrient-dense diet and for chronic disease prevention; however, in both highly developed and developing countries intakes are typically lower than recommended levels, including intakes among pregnant women. ${ }^{5,17-20}$ Low intake of fruits and vegetables may be attributed to a variety of factors such as taste, cost, familiarity and habit, availability, and time for preparation. ${ }^{21-24}$ Conflicting results regarding associations of maternal exposure to organophosphorus insecticides used on fruits and vegetables and birth weight outcomes also could cause some women to limit consumption of fruits and vegetables during pregnancy. ${ }^{25,26}$

A better understanding of the association between intake of fruits and vegetables during pregnancy and birth weight may have important implications for refining and promoting dietary guidance for pregnant women. The authors are not aware of a comprehensive review of associations between maternal consumption of fruits and vegetables during pregnancy and birth weight or delivery of an SGA infant. The purpose of this study was to systematically review the literature on associations of maternal consumption of fruits and vegetables with infant birth weight and risk for SGA births.

\section{Methods}

\section{Literature search}

The PubMed and EMBASE databases were searched to identify studies that examined associations between fruit and vegetable consumption during pregnancy and infant birth weight or risk for SGA births. The search in PubMed was conducted through June 1, 2014, with the terms: ("Birth Weight"[Mesh] OR "Infant, Small for Gestational Age"[Mesh] or birthweight or "birth weight"
OR "small for gestational age" or "small-for-gestationalage") AND ("Diet"[Mesh] or fruit or vegetable or fruits or vegetables or "Fruit"[Mesh] OR "Vegetables"[Mesh]) AND ([“Pregnancy" $\{$ Mesh $\}]$ or pregnancy or pregnant or maternal). The search was limited to human studies and English language papers with no lower date limit, yielding a total of 1,024 publications. The EMBASE database also was searched using the search string: 'fruit'/exp OR 'fruit' OR 'fruit'/exp OR fruit OR 'vegetable'/exp OR 'vegetable' OR 'vegetable'/exp OR vegetable OR fruits OR 'vegetables'/exp OR 'vegetables' OR 'vegetables'/exp OR vegetables AND ('birthweight'/exp OR 'birthweight' OR 'birthweight'/exp OR birthweight OR 'birth weight'/exp OR 'birth weight' OR fetal AND ('growth'/exp OR 'growth' OR 'growth'/ exp OR growth) OR 'infant, small for gestational age'/exp OR 'infant, small for gestational age' OR sga OR 'small for gestational age'/exp OR 'small for gestational age' OR 'small-for-gestational-age'/exp OR 'small-for-gestationalage'). The search was limited to human studies and English language papers, yielding a total of 389 publications.

Titles and abstracts of the identified papers were screened and 42 potentially relevant papers were identified. Full papers were then reviewed to determine relevance. Observational and intervention studies were eligible for inclusion if the study population was generally healthy women and the study reported original data on the association of consumption of fruit and/or vegetables during pregnancy (collected prospectively or retrospectively) and infant birth weight or risk for an SGA birth. There were no limits placed on location of the study populations. Reasons for exclusion included: examined association between birth weight and a dietary pattern containing fruits and/or vegetables (eg, the Mediterranean Diet) rather than specifically consumption of fruits and/or vegetables; data on associations between fruit and vegetable consumption and birth weight were not provided in text; or non-peer reviewed findings such as review papers or abstracts. The electronic searches were supplemented with manual searches of bibliographies of all relevant papers and pertinent review articles. An independent examination of all potentially relevant papers was completed by two reviewers (MMM and KMS); discrepancies were resolved by consensus or by a third author (NS). The search process is summarized in Figure 1. A total of eleven publications were determined to be eligible for inclusion in the review.

\section{Data extraction}

The identified papers were reviewed and key data were extracted including: study population, study design, location, 


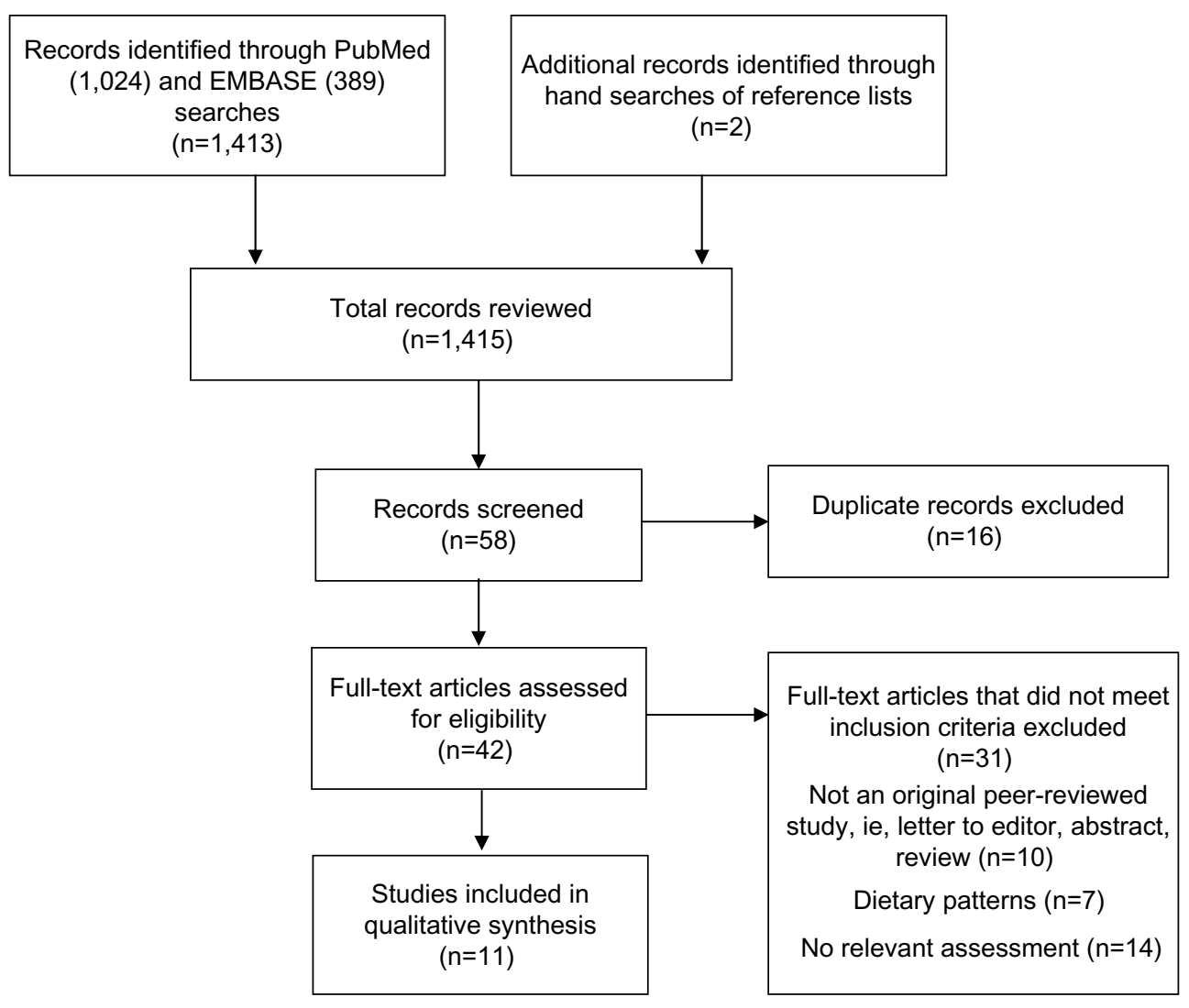

Figure I Flow diagram of study identification.

when the exposure assessment was administered (eg, at 15 weeks' gestation or retrospectively following birth), the period of pregnancy covered by the exposure assessment, method of exposure assessment, specific fruits and vegetables included in the exposure assessment, mean intake of fruits and vegetables, method of analysis of the association between maternal intake of fruits and vegetables and birth weight or risk for SGA, potential confounders in the analyses, and the study findings. Authors were contacted for clarifying information on reported associations of fruit and vegetable intake and birth weight or risk for SGA birth. Study quality was assessed using established criteria for evaluation of cohort and case-control studies. ${ }^{27}$

\section{Results}

\section{Studies included in the analysis}

Eleven studies of the association between maternal fruits and vegetables consumption during pregnancy and infant birth weight or risk for SGA birth were identified. ${ }^{14-16,28-35}$ Pregnant women residing in countries within Europe or Australasia ranking very high on metrics of human development as defined by the Human Development Index (HDI) comprised the study population of seven studies. ${ }^{15,16,28-32,36}$
The remaining four studies were conducted among pregnant women living in countries with medium (India and Egypt) or high (Malaysia) rankings on the HDI. ${ }^{14,33-35}$ The eleven identified studies were all observational and included six prospective cohort studies, ${ }^{14,15,28,29,34,35}$ three retrospective cohort studies, ${ }^{30,32,33}$ and two case-control studies. ${ }^{16,31}$ No intervention study exclusively targeting intake of fruits and/or vegetables during pregnancy was identified.

Table 1 presents a summary of the study population, period of time during pregnancy covered by the exposure assessment, and outcome classification in each study. The studies assessed exposures using food frequency questionnaires (FFQs) and interview techniques. Exposures to vegetables were categorized in the studies as vegetables, green leafy vegetables, non-green leafy vegetables, green vegetables, and carrots; exposures to fruits were categorized as fruit (which may have included juice) or fruit and juice. The types of vegetables and fruits captured in the exposure assessments are summarized in Table 1 by broad category, namely green leafy, brassica, red/orange, bean/pea, starchy, and other vegetables; and berries, citrus, pome, stone, tropical, melon, and fruit juices. Vegetable and fruit exposures were quantified in the studies as number of servings per day 


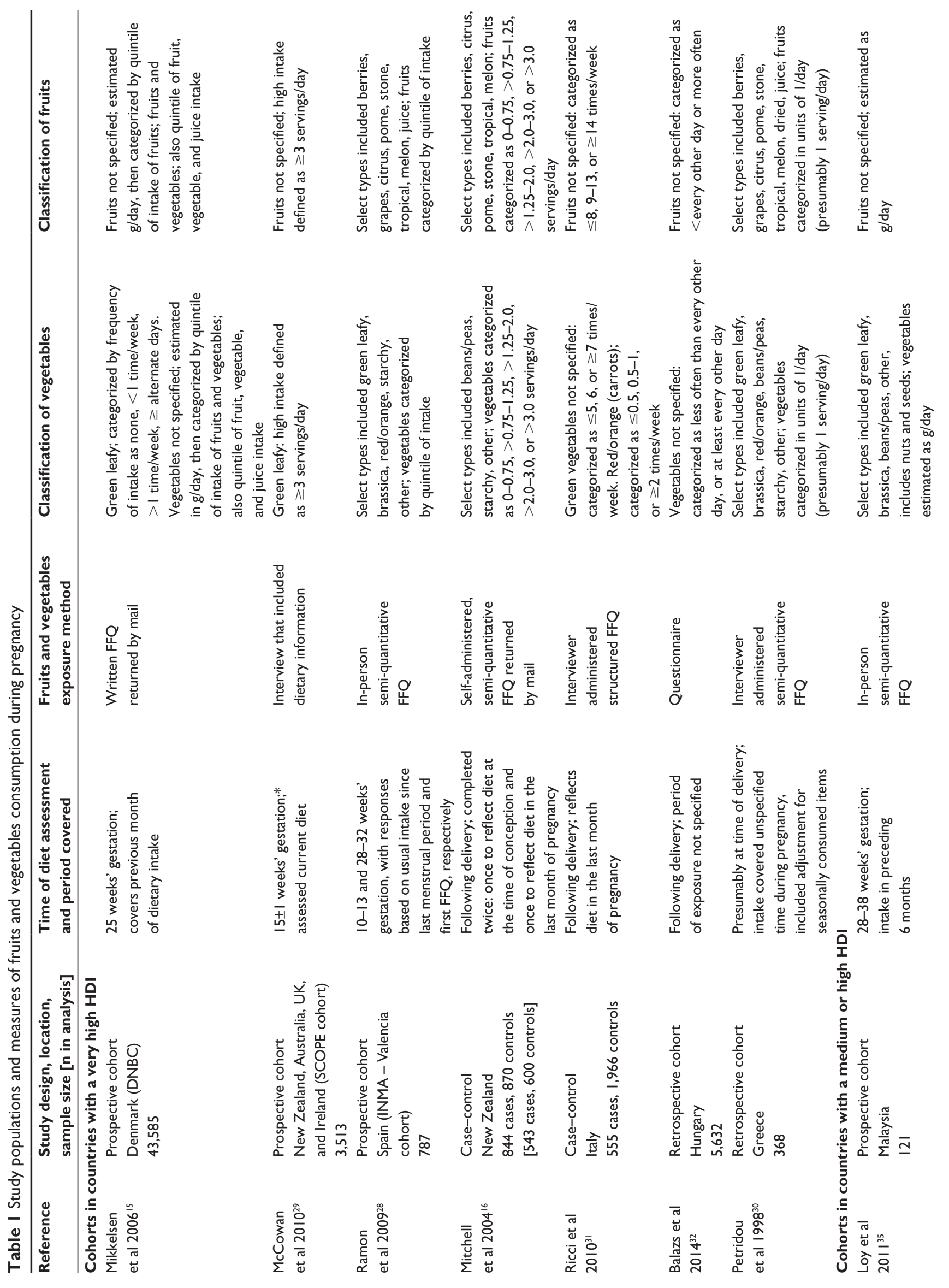




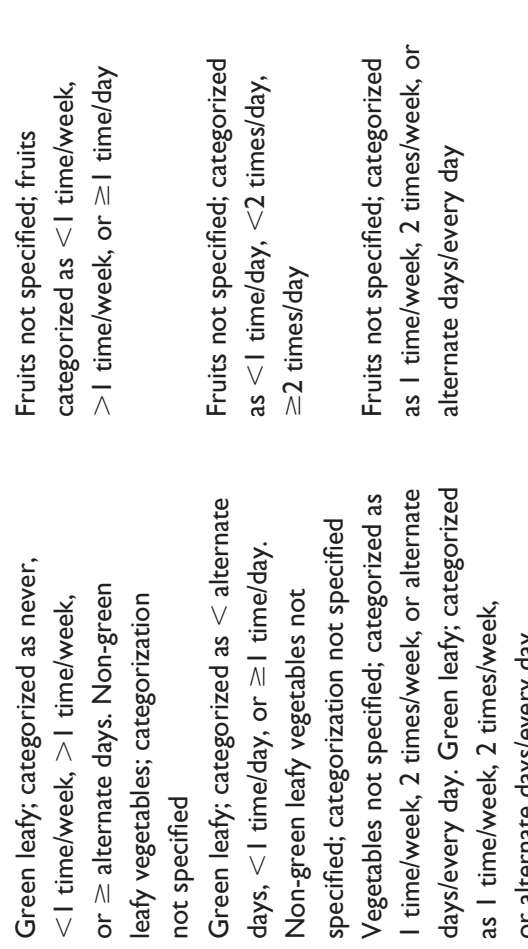

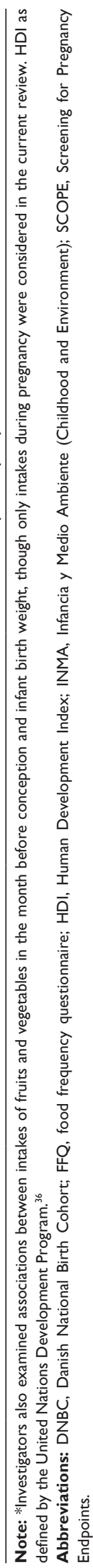

(continuous or categorical), frequency of intake (categorical), $\mathrm{g} /$ day, or quintiles of intake.

Confounders controlled for in the statistical analyses varied among the studies; they are summarized by study in Table 2. These factors included modifiable and nonmodifiable factors as well as maternal and infant characteristics. The confounders most frequently controlled for in the analyses included parity, maternal age, pre-pregnancy body mass index or body weight, smoking, infant sex, maternal height, and one or more indicators of socioeconomic status.

\section{Dietary intake and birth weight or SGA status}

Associations between maternal intake of vegetables or fruits during pregnancy and birth weight or SGA births for cohorts residing in countries with a very high HDI are shown in Table 3; associations for cohorts living in countries with a medium or high HDI are shown in Table 4.

\section{Maternal vegetables intake and birth weight or SGA status}

In a prospective study following a cohort of 787 pregnant women in Spain, a country with a very high HDI ranking, maternal intake of vegetables during the first trimester of pregnancy was associated with increased infant birth weight, with newborns of women in the first and second quintiles of vegetable consumption weighing less than newborns of infants in the fourth quintile $(148.2 \pm 46.6$ and $132.5 \pm 45.1 \mathrm{~g}$, respectively; $P<0.01$ ) (Table 3 ). ${ }^{28}$ In the same study, women in the lowest quintile of vegetable intake during the first trimester of pregnancy, though not the third trimester, were at significantly higher risk of delivering an SGA baby than women in the highest quintile of vegetable intake in a model using fruit and vegetable variables adjusted for energy intake (adjusted odds ratio 3.1; 95\% confidence interval [CI] $1.4-6.9 ; P=0.01) .{ }^{28}$ In addition to energy intake, confounding variables in the analysis of risk for SGA birth included maternal age, pre-pregnant weight, maternal height, weight gain, parity, smoking during pregnancy, alcohol consumption, caffeine consumption, working, country of origin, and residence. Mean vegetable intake in the lowest quintile of intake was $72.8 \mathrm{~g} /$ day, which is equivalent to slightly less than one serving daily assuming approximately $80 \mathrm{~g}$ per serving. Results from this study provide limited evidence that increased vegetable intake early in pregnancy may be associated with reduced risk for SGA birth, particularly among women consuming on average no more than a serving of vegetables per day. 


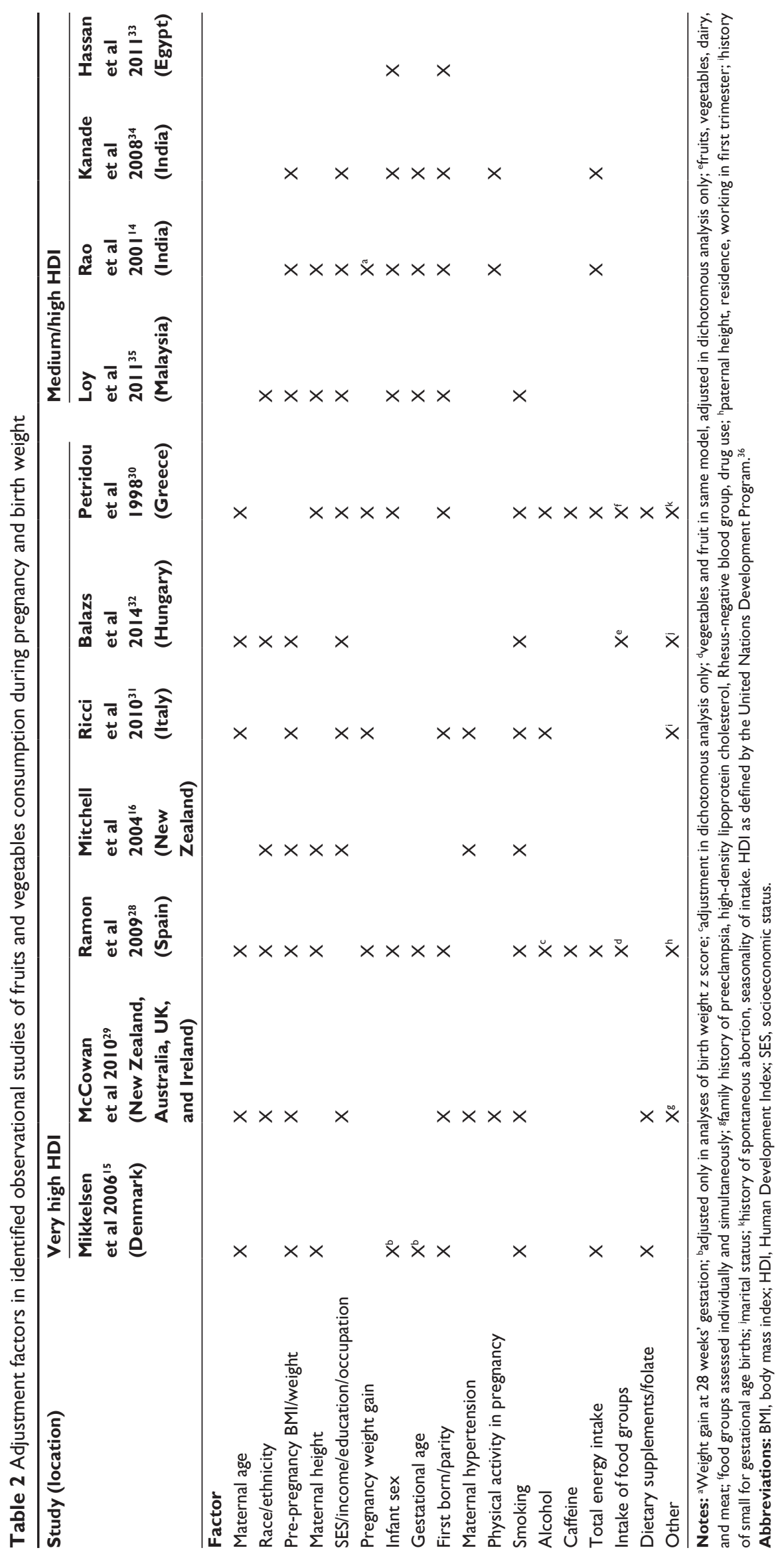




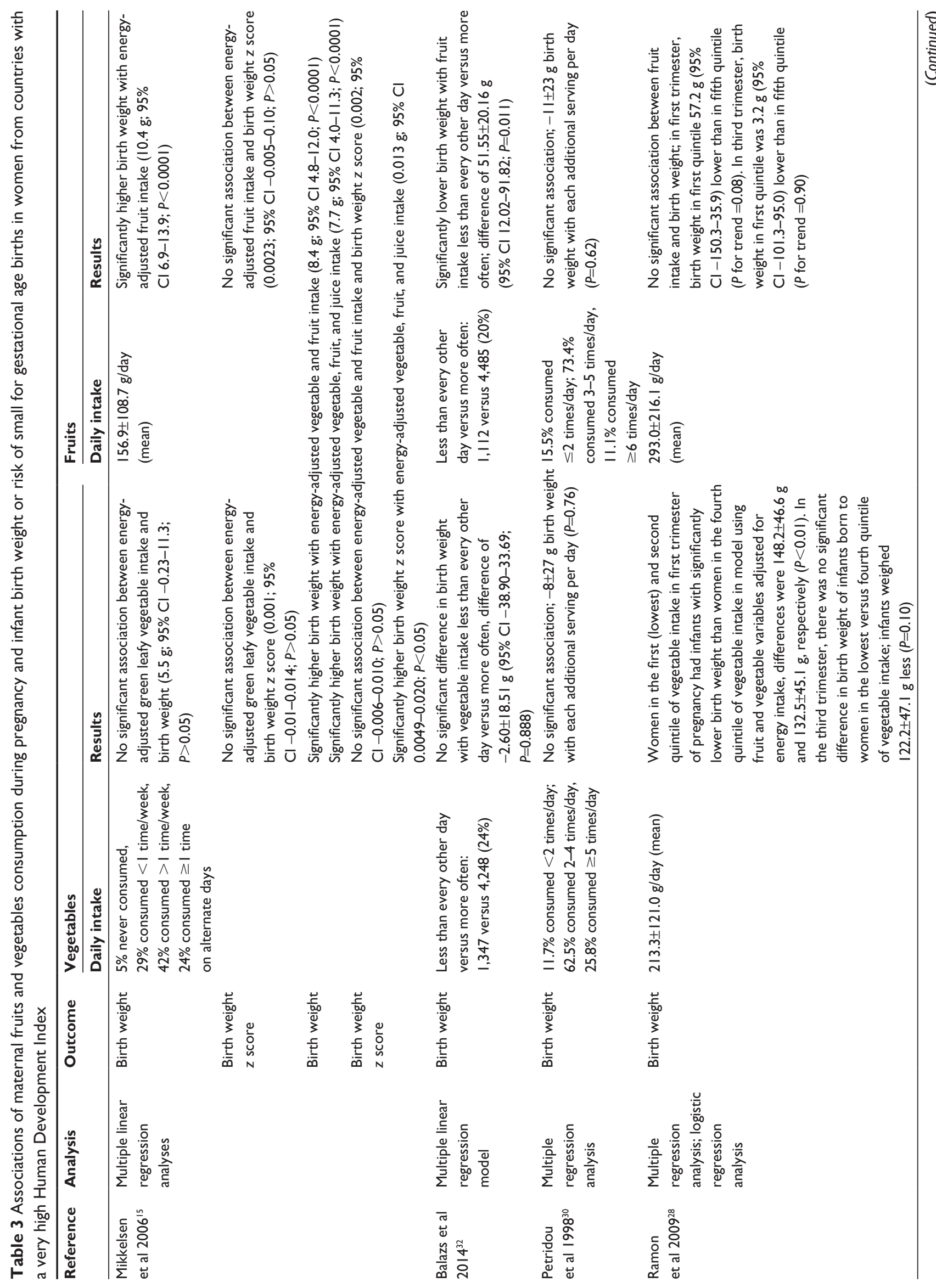




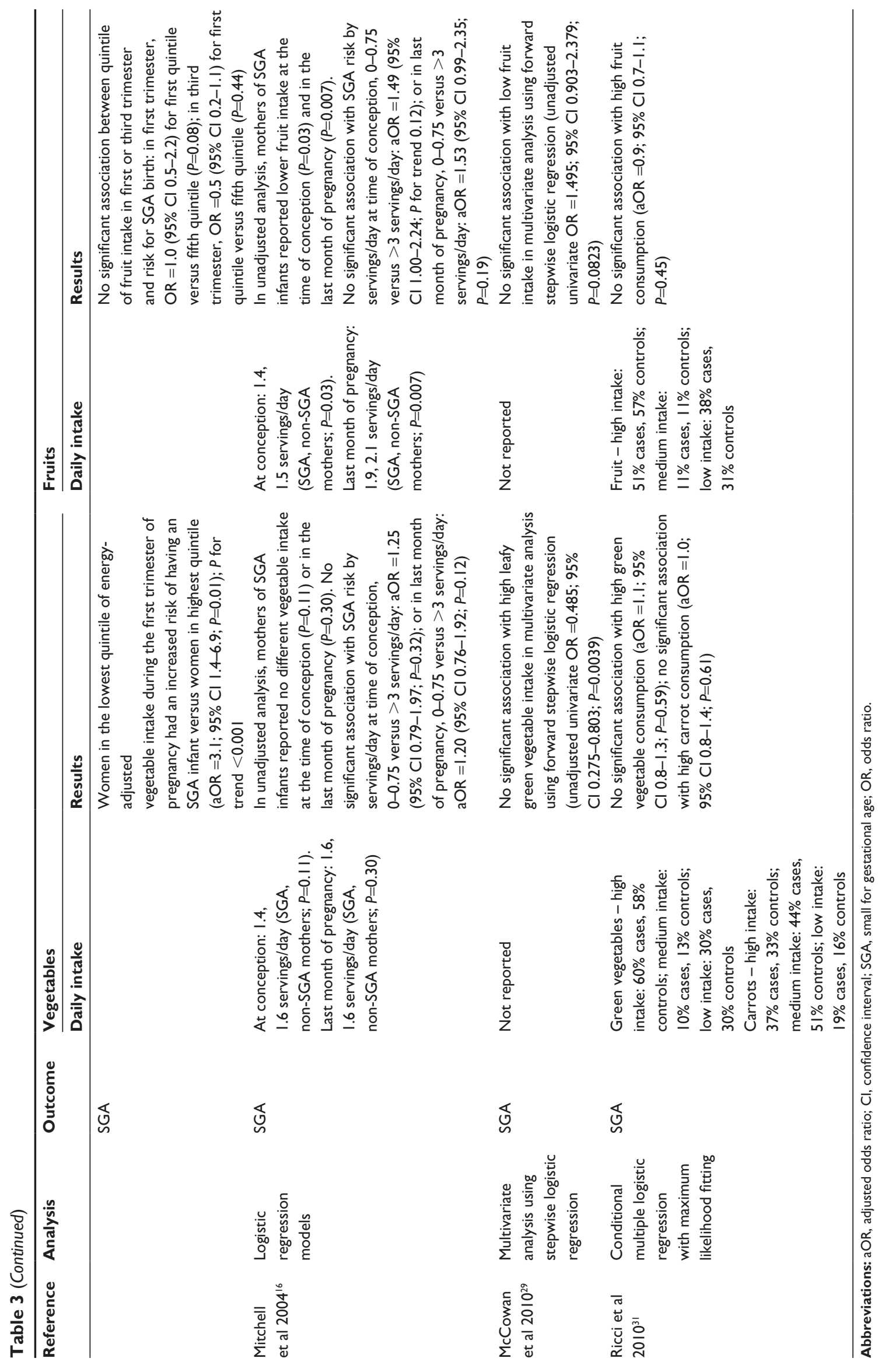




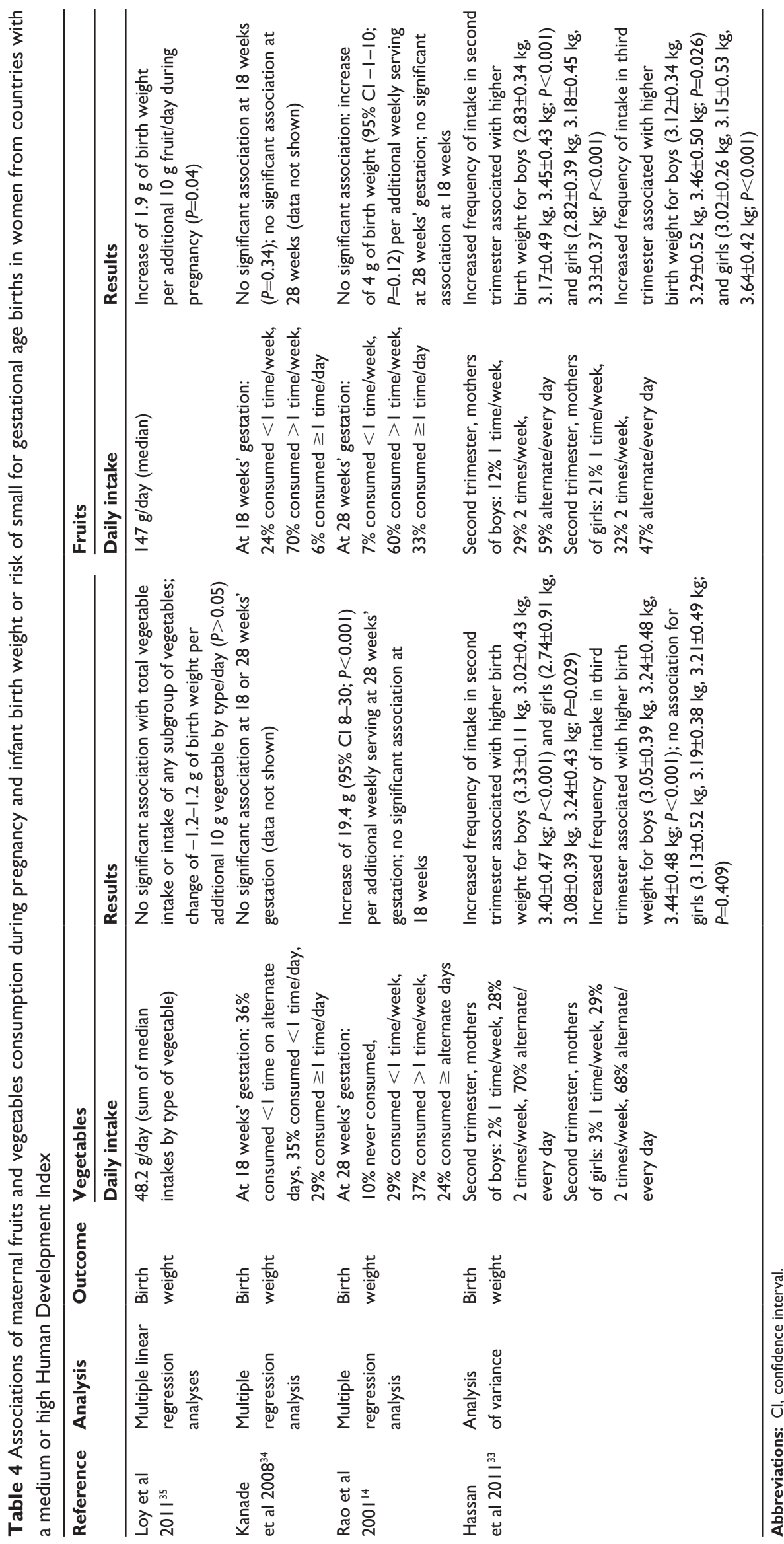


In the remaining six out of the seven studies conducted on populations of women from countries with very high HDI rankings, analysis of the relationship between maternal intake of vegetables by women and birth weight failed to demonstrate a significant association. ${ }^{15,16,29-32}$ In a large cohort of pregnant women in Denmark, intake of green leafy vegetables reported at 25 weeks' gestation was associated with a $6.1 \mathrm{~g}$ (95\% CI $0.35-11.8 ; P<0.05)$ increase in birth weight per categorical increase in consumption, but the difference was no longer observed when intakes were energy-adjusted or in analyses of birth weight $\mathrm{z}$ scores. ${ }^{15} \mathrm{In}$ a prospective study conducted in a cohort of 3,513 women from highly developed areas (New Zealand, Australia, the UK, and Ireland), namely the Screening for Pregnancy Endpoints (SCOPE) study, consumption of leafy green vegetables at 15 weeks' gestation was associated with reduced risk for SGA in an unadjusted analysis (odds ratio 0.485 ; 95\% CI $0.275-0.803$; $P=0.0039$ ), though intake of green leafy vegetables during pregnancy was not a significant variable for SGA risk in the final adjusted model developed using forward stepwise logistic regression. ${ }^{29}$ Pre-pregnancy intakes of leafy green vegetables (and also intakes of fruits) were, however, determined to be significantly associated with SGA risk.

Intake of vegetables by women during pregnancy was positively associated with infant birth weight in two of the four studies conducted in countries with lower HDI rankings (Table 4). ${ }^{14,33}$ In a population of rural, undernourished women in India, each additional serving of green leafy vegetables at 28 weeks' (but not 18 weeks') gestation was associated with an increase in birth weight of $19.4 \mathrm{~g} \mathrm{(95 \%} \mathrm{CI} \mathrm{8-30} \mathrm{g;}$ $P<0.001) .{ }^{14}$ Among pregnant women in Egypt, increased frequency of consumption of green leafy vegetables was associated with higher birth weight in analyses controlling for infant sex and parity; women consuming green leafy vegetables on alternate days or every day in the second trimester of pregnancy delivered boys weighing on average $380 \mathrm{~g}$ more than women consuming green leafy vegetables twice a week $(P<0.001)$, while women consuming green leafy vegetables on alternate days or every day delivered girls weighing on average $500 \mathrm{~g}$ more than women consuming vegetables only once a week $(P<0.029) .{ }^{33}$ In the same study, frequency of green leafy vegetable intake during the third trimester of pregnancy also was associated with birth weight of boys $(P<0.001)$, though a significant association was not observed for girls $(P=0.409) .{ }^{33}$ Given the lack of adjustment for potential confounders other than parity and infant's sex, this study provides extremely weak evidence of an association between consumption of vegetables during pregnancy and birth weight. No significant association between consumption of vegetables during pregnancy and birth weight was observed in prospective cohorts in Malaysia or urban areas of India. ${ }^{34,35}$ No studies in countries with a very high HDI or in less developed countries reported a significant inverse association between maternal intake of vegetables and birth weight or SGA births.

\section{Maternal fruits intake and birth weight or SGA status}

Maternal intake of fruits during pregnancy was associated with increased infant birth weight in two studies of women from countries with very high HDI rankings (Table 3). ${ }^{15,32}$ In a large cohort of pregnant women in Denmark, energyadjusted intake of fruits in the second trimester of pregnancy (measured at 25 weeks' gestation) was associated with a $10.4 \mathrm{~g}$ higher birth weight per categorical increase $(95 \%$ CI 6.9-13.9; $P<0.0001)$; however, no significant association was observed between maternal fruits intake and birth weight $\mathrm{z}$ score, which included adjustments for infant sex and gestational age $(0.0023 ; 95 \% \mathrm{CI}-0.005-0.10 ; P>0.05){ }^{15}$ In a retrospective study in 5,632 Roma and non-Roma women in Hungary, women consuming fruits less frequently than every other day delivered infants with a birth weight $51.55 \pm 20.16 \mathrm{~g}$ lower $(95 \%$ CI $12.02-91.82 ; P=0.011)$ than women consuming fruits more often. ${ }^{32}$ It is difficult to draw firm conclusions from this retrospective study as women were interviewed in the calendar year following the birth of a term infant and very limited information was provided on the dietary assessment.

In the remaining five studies conducted in cohorts of women from countries with very high HDI rankings, no significant association between maternal intake of fruits during pregnancy and birth weight or risk for SGA birth was observed. ${ }^{16,28-31}$ In a case-control study of women in Italy, women delivering SGA infants consumed fewer fruits compared to women delivering healthy term infants with a normal weight (10.4 versus $11.4 \mathrm{~g} /$ day; $P<0.001$ ); fruit intake was not, however, significantly associated with risk for SGA birth (adjusted odds ratio $0.9 ; 95 \% \mathrm{CI} 0.7-1.1 ; P=0.45$ ). ${ }^{31}$

Maternal intake of fruits during pregnancy was positively associated with birth weight in two of the four studies conducted in cohorts of women from countries with medium or high HDI rankings (Table 4). ${ }^{33,35}$ In a small prospective cohort study of 121 women, each additional $10 \mathrm{~g}$ of fruits intake per day during pregnancy among healthy Malaysian women was associated with an increase of $1.9 \mathrm{~g}$ of birth weight $(P=0.04) .{ }^{35}$ Among women in Egypt delivering boys, increased frequency of consumption of fruits during 
the second and third trimesters was associated with higher birth weight $(P<0.001$ and $P<0.026 ; 620 \mathrm{~g}$ and $340 \mathrm{~g}$ for alternate days/every day versus once a week in second and third trimesters, respectively). Among women delivering girls, increased frequency of consumption of fruits during the second trimester and third trimester was associated with higher birth weight $(P<0.001$ in each trimester; $510 \mathrm{~g}$ and $620 \mathrm{~g}$ for alternate days/every day versus once a week in second and third trimesters, respectively). ${ }^{33}$ As previously noted, findings from the study in Egypt are extremely weak given the lack of adjustment for potential confounders other than parity and infant's sex. No studies in countries with a very high HDI or in less developed countries reported a significant association between decreased birth weight or an increased risk of delivering an SGA infant with increased consumption of fruits.

\section{Maternal combined fruits and vegetables intake and birth weight/SGA status}

In addition to studying associations between consumption of green leafy vegetables or fruit intake during pregnancy and birth weight, Mikkelsen et al also examined the association of combined fruits and vegetables intake and birth weight in the Danish National Birth Cohort (Table 3). ${ }^{15}$ In this study of 43,585 pregnant women, the largest study identified in this review, each energy-adjusted quintile of increased fruits and vegetables (combined) intake mid-pregnancy (approximately weeks 21-25) was associated with an increase in birth weight of 8.4 g per quintile $(95 \%$ CI $4.8-12.0 ; P<0.0001)$ and energy-adjusted intake of fruit, vegetables, and juice (combined) was associated with an increase in birth weight of $7.7 \mathrm{~g}$ per quintile (95\% CI 4.0-11.3; $P<0.0001)$. As previously noted, energy-adjusted consumption of fruits was associated with increased birth weight, though consumption of green leafy vegetables was not significantly associated with birth weight in this study; therefore, it appears that increased consumption of fruits may largely account for the observed associations between combined fruits and vegetables intake and birth weight. Intakes of fruits and vegetables were estimated from responses to a validated food frequency questionnaire and confounding variables in the analyses included use of dietary supplements, smoking, maternal height, pre-pregnancy weight, paternal height, parity, and maternal age as well as energy intake. With an additional adjustment for gestational age and sex in the assessment of associations with birth weight z scores, only increased intake of fruits, vegetables, and juice combined remained significantly associated with a higher birth weight $\mathrm{z}$ score - birth weight $\mathrm{z}$ scores increased 0.013
(95\% CI 0.0049-0.020; $P<0.01)$. The authors speculated that the stronger associations of diet with birth weight than with birth weight $\mathrm{z}$ score may reflect an association with both birth weight and length of gestation. The investigators also noted that the mean birth weight observed in the Danish National Birth Cohort was $200 \mathrm{~g}$ higher than in the overall Danish population; the implications of this higher birth weight in the analysis of associations between diet and birth weight is unknown. An examination of associations between maternal intake of fruits and vegetables and risk for SGA birth was not reported. The overall findings from this study provide limited evidence of an association between mid-pregnancy intake of fruits and vegetables and birth weight.

\section{Discussion}

In this systematic review of observational studies of associations between maternal intake of fruits and vegetables during pregnancy and birth weight outcomes, eleven studies were identified, including seven cohorts from countries with a very high HDI ranking and four cohorts from countries with a medium or high HDI ranking. Overall, limited evidence of a positive association between fruit and vegetable consumption during pregnancy and birth weight was identified, as the majority of studies in cohorts of women in highly developed areas failed to demonstrate any association between consumption of fruits or vegetables during pregnancy and birth weight or risk for SGA birth. A significant association between fruits or vegetables and birth weight was reported in two prospective studies conducted with women from countries with a medium or high HDI ranking. None of the studies reviewed reported a significantly increased risk for SGA or a significantly lower birth weight with increasing consumption of fruits or vegetables.

The available studies of cohorts in highly developed areas provided limited evidence suggesting that increased consumption of fruits and vegetables in mid-pregnancy driven primarily by fruit intake - may be associated with increased birth weight, and limited evidence suggesting that increased consumption of vegetables in early pregnancy by women with very low intakes may be associated with a reduced risk for SGA birth. Among women in less developed countries, limited evidence suggests that increased consumption of vegetables or fruits may be associated with higher infant birth weights. Increased consumption of fruits and vegetables combined among healthy women living in a highly developed area was associated with an increase of approximately $8 \mathrm{~g}$ in birth weight per categorical increase, while increased consumption of vegetables among women 
from a country with a medium HDI ranking was associated with a categorical increase of $19.4 \mathrm{~g}$ in birth weight. Although the available evidence is extremely limited, the data suggest that the association between increased consumption of fruits and vegetables may be stronger among cohorts from less highly developed areas who are less well nourished than the cohorts in very highly developed areas.

Several mechanisms for an association between increased consumption of fruits and vegetables and birth weight can be hypothesized. Fruits and vegetables are key sources of several important nutrients, including potassium, magnesium, dietary fiber, folate, and vitamins $\mathrm{A}$ and $\mathrm{C} .{ }^{13} \mathrm{~A}$ diet rich in fruits and vegetables in non-pregnant women is known to decrease blood pressure, and hypertension is a major risk factor for restricted growth. ${ }^{1,37}$ Some micronutrients contained in fruits and vegetables may also contribute to optimal immune and placental functions, which are important for fetal growth. ${ }^{38,39}$ Alternatively, increased fruit and vegetable intake may simply be a marker for healthier dietary patterns or a healthier lifestyle that could not be fully adjusted in these observational studies.

Analyses of dietary patterns using factor or cluster analyses provide insights on potential associations between the overall quality of the maternal diet and birth weight. In a large sample of Danish women, consumption of a "health conscious diet" (vegetables, fruits, poultry, and fish) during pregnancy was associated with a $26 \%$ decrease $(95 \%$ CI 14-36\%) in the risk of delivering an SGA infant compared to a "Western diet" (red meat, processed meat, and high-fat dairy). ${ }^{2}$ In New Zealand, increased adherence to elements of a traditional diet (lamb, root vegetables, peas, gravy, and meat dishes) was associated with a decreased risk of delivering an SGA infant. ${ }^{8}$ In a smaller study of Japanese women, consumption of a "wheat products" dietary pattern was associated with a 5.2-fold increased risk for delivering an SGA infant (95\% CI 1.1-24.4) compared to a "rice, fish, and vegetables" pattern. ${ }^{6}$ Increased consumption of fruits and vegetables appears to be a common factor of several of these favorable dietary patterns.

Variability across the studies in the period of dietary intake during pregnancy captured by the assessments, the categorization of fruit and vegetable intakes, and the specific outcome examined (birth weight or risk for SGA birth) make it difficult to make direct comparisons across studies grouped by HDI. Among the studies conducted in cohorts of women from highly developed countries, three studies assessed intakes late in pregnancy based on an assessment completed retrospectively after birth ${ }^{30-32}$ and one retrospective assessment captured intakes both late in pregnancy and at the time of conception; ${ }^{16}$ two prospective studies captured intakes in approximately the first trimester of pregnancy; ${ }^{28,29}$ and two prospective studies captured intakes mid-pregnancy. ${ }^{15,28}$ The types of vegetables captured in the exposure assessments included only green leafy vegetables in two studies among the studies conducted in cohorts of women from highly developed countries, ${ }^{15,29}$ unspecified green vegetables and carrots in one study; ${ }^{31}$ and all vegetables (based on reported intake of all vegetables or reported intakes of several specific types) in four studies. ${ }^{16,28,30,32}$ In all studies, only one category of fruit was examined, and fruit intake may have been captured based on reported intake of all fruits or reported intakes of several specific types. Among the studies conducted in cohorts of women from highly developed countries, three studies examined associations between maternal fruit or vegetable intake and birth weight, ${ }^{15,30,32}$ three examined associations between maternal intake and risk for SGA birth, ${ }^{16,29,31}$ and one study examined both associations. ${ }^{28}$ All studies were adjusted for some potential confounding factors, though the specific factors varied across the studies. In all studies, additional factors not considered in the analyses may have influenced the findings.

Another limitation of the present review is that not all studies took into account the amount of fruits and vegetables consumed, ${ }^{14,31-34}$ which could be an important source of bias towards the null hypothesis. Also, none of the studies reviewed explored whether the production of the fruits and vegetables was organic or conventional. Maternal exposure to organophosphorus insecticides has been associated with low birth weight in one study, ${ }^{25}$ but not in another. ${ }^{26}$ However, since it is unlikely that the fruits and vegetables consumed in the reviewed studies were mostly organic, the results of this review suggest that a possible association between pesticides and birth weight should not deter pregnant women from consuming fruits and vegetables even if they cannot afford organically grown products. Similarly, none of the studies reviewed explored if the association between fruits and vegetables intake and birth weight was different between fresh, canned, or frozen fruits and vegetables. Therefore, unless other data become available, all of these options should be considered to increase intake when resources and access are limited.

\section{Conclusion}

In this systematic review of observational studies, limited though inconclusive evidence of a protective effect of increased consumption of vegetables on risk for SGA birth 
and increased consumption of fruits and vegetables and increased birth weight among women from highly developed countries was identified. Among women in less developed countries, limited evidence suggests that increased consumption of vegetables or fruits may be associated with higher infant birth weight. No studies suggested that an increased intake of fruits or vegetables was associated with significantly lower birth weight or increased risk for SGA. Fruits and vegetables are rich sources of many important vitamins and minerals as well as dietary fiber, and dietary guidance for all individuals encourages daily consumption of fruits and vegetables as part of a nutrient-dense diet. Additional wellconducted observational or intervention studies are warranted to better understand the specific role of fruits and vegetables during pregnancy on birth weight. The available evidence, however, supports maternal consumption of a variety of fruits and vegetables as part of a balanced diet.

\section{Acknowledgments}

Funding for this research was provided by CropLife America. The study sponsor had no role in the study design; collection, analysis, or interpretation of data; or writing of the manuscript. Nicholas Settler was affiliated with Exponent, Inc. at the time of this analysis, but is currently with The Lewin Group.

\section{Authors' contributions}

MMM and NS contributed to the conception and design; literature review, analysis, and interpretation; and drafting and critical review of the manuscript. KMS contributed to the literature review, data extraction and interpretation, and revision of the manuscript. RR conceived the study idea and contributed to the design, interpretation, and critical review of the manuscript. All authors have given final approval of the version to be published and agree to be accountable for all aspects of the work.

\section{Disclosure}

The authors report no conflicts of interest in this work.

\section{References}

1. World Health Organization. Promoting Optimal Fetal Development: Report of a Technical Consultation. Geneva: World Health Organization; 2006. Available from: http://www.who.int/nutrition/topics/fetal_dev_ report_EN.pdf. Accessed December 27, 2012.

2. Knudsen VK, Orozova-Bekkevold IM, Mikkelsen TB, Wolff S, Olsen SF. Major dietary patterns in pregnancy and fetal growth. Eur J Clin Nutr. 2008;62(4):463-470.

3. Yang Z, Huffman SL. Review of fortified food and beverage products for pregnant and lactating women and their impact on nutritional status. Matern Child Nutr. 2011;7(Suppl 3):19-43.
4. Haider BA, Yakoob MY, Bhutta ZA. Effect of multiple micronutrient supplementation during pregnancy on maternal and birth outcomes. BMC Public Health. 2011;11(Suppl 3):S19.

5. Rodriguez-Bernal CL, Rebagliato M, Iniguez C, et al. Diet quality in early pregnancy and its effects on fetal growth outcomes: the Infancia y Medio Ambiente (Childhood and Environment) Mother and Child Cohort Study in Spain. Am J Clin Nutr. 2010;91(6):1659-1666.

6. Okubo H, Miyake Y, Sasaki S, et al. Maternal dietary patterns in pregnancy and fetal growth in Japan: the Osaka Maternal and Child Health Study. Br J Nutr. 2012;107(10):1526-1533.

7. Wolff $\mathrm{CB}$, Wolff HK. Maternal eating patterns and birth weight of Mexican American infants. Nutr Health. 1995;10(2):121-134.

8. Thompson JM, Wall C, Becroft DM, Robinson E, Wild CJ, Mitchell EA. Maternal dietary patterns in pregnancy and the association with small-for-gestational-age infants. Br J Nutr. 2010;103(11): $1665-1673$.

9. Mannion CA, Gray-Donald K, Koski KG. Association of low intake of milk and vitamin D during pregnancy with decreased birth weight. CMAJ. 2006;174(9):1273-1277.

10. Olsen SF, Halldorsson TI, Willett WC, et al. Milk consumption during pregnancy is associated with increased infant size at birth: prospective cohort study. Am J Clin Nutr. 2007;86(4):1104-1110.

11. Rogers I, Emmett P, Ness A, Golding J. Maternal fish intake in late pregnancy and the frequency of low birth weight and intrauterine growth retardation in a cohort of British infants. J Epidemiol Community Health. 2004;58(6):486-492.

12. Olsen SF, Secher NJ. Low consumption of seafood in early pregnancy as a risk factor for preterm delivery: prospective cohort study. $B M J$. 2002;324(7335):447.

13. Liu RH. Health-promoting components of fruits and vegetables in the diet. Adv Nutr. 2013;4(3):384S-392S.

14. Rao S, Yajnik CS, Kanade A, et al. Intake of micronutrient-rich foods in rural Indian mothers is associated with the size of their babies at birth: Pune Maternal Nutrition Study. J Nutr. 2001;131(4): $1217-1224$

15. Mikkelsen TB, Osler M, Orozova-Bekkevold I, Knudsen VK, Olsen SF. Association between fruit and vegetable consumption and birth weight: a prospective study among 43,585 Danish women. Scand J Public Health. 2006;34(6):616-622.

16. Mitchell EA, Robinson E, Clark PM, et al. Maternal nutritional risk factors for small for gestational age babies in a developed country: a case-control study. Arch Dis Child Fetal Neonatal Ed. 2004;89(5): F431-F435.

17. Krebs-Smith SM, Guenther PM, Subar AF, Kirkpatrick SI, Dodd KW. Americans do not meet federal dietary recommendations. J Nutr. 2010;140(10):1832-1838.

18. Hall JN, Moore S, Harper SB, Lynch JW. Global variability in fruit and vegetable consumption. Am J Prev Med. 2009;36(5):402.e5-409.e5.

19. Crozier SR, Robinson SM, Borland SE, et al. Do women change their health behaviours in pregnancy? Findings from the Southampton Women's Survey. Paediatr Perinat Epidemiol. 2009;23(5): 446-453.

20. Fowler JK, Evers SE, Campbell MK. Inadequate dietary intakes among pregnant women. Can J Diet Pract Res. 2012;73(2):72-77.

21. Schatzer M, Rust P, Elmadfa I. Fruit and vegetable intake in Austrian adults: intake frequency, serving sizes, reasons for and barriers to consumption, and potential for increasing consumption. Public Health Nutr. 2010;13(4):480-487.

22. Haynes-Maslow L, Parsons SE, Wheeler SB, Leone LA. A qualitative study of perceived barriers to fruit and vegetable consumption among low-income populations, North Carolina, 2011. Prev Chronic Dis. 2013;10:E34.

23. Drewnowski A, Gomez-Carneros C. Bitter taste, phytonutrients, and the consumer: a review. Am J Clin Nutr. 2000;72(6):1424-1435.

24. Pollard J, Kirk SF, Cade JE. Factors affecting food choice in relation to fruit and vegetable intake: a review. Nutr Res Rev. 2002;15(2): 373-387. 
25. Rauch SA, Braun JM, Barr DB, et al. Associations of prenatal exposure to organophosphate pesticide metabolites with gestational age and birth weight. Environ Health Perspect. 2012;120(7):1055-1060.

26. Eskenazi B, Harley K, Bradman A, et al. Association of in utero organophosphate pesticide exposure and fetal growth and length of gestation in an agricultural population. Environ Health Perspect. 2004;112(10): 1116-1124.

27. United States Preventive Services Task Force. US Preventive Services Task Force Procedure Manual. Rockville, MD: Agency for Healthcare Research and Quality; 2008. Available from: http://www. uspreventiveservicestaskforce.org/uspstf08/methods/procmanual.pdf. Accessed December 27, 2012.

28. Ramon R, Ballester F, Iniguez C, et al. Vegetable but not fruit intake during pregnancy is associated with newborn anthropometric measures. J Nutr. 2009;139(3):561-567.

29. McCowan LM, Roberts CT, Dekker GA, et al. Risk factors for smallfor-gestational-age infants by customised birthweight centiles: data from an international prospective cohort study. BJOG. 2010;117(13): 1599-1607.

30. Petridou E, Stoikidou M, Diamantopoulou M, Mera E, Dessypris N, Trichopoulos D. Diet during pregnancy in relation to birthweight in healthy singletons. Child Care Health Dev. 1998;24(3):229-242.

31. Ricci E, Chiaffarino F, Cipriani S, Malvezzi M, Parazzini F. Diet in pregnancy and risk of small for gestational age birth: results from a retrospective case-control study in Italy. Matern Child Nutr. 2010;6(4): 297-305.

32. Balazs P, Rakoczi I, Grenczer A, Foley KL. Birth-weight differences of Roma and non-Roma neonates - public health implications from a population-based study in Hungary. Cent Eur J Public Health. 2014;22(1):24-28.
33. Hassan NE, Shalaan AH, El-Masry SA. Relationship between maternal characteristics and neonatal birth size in Egypt. East Mediterr Health J. 2011;17(4):281-289.

34. Kanade AN, Rao S, Kelkar RS, Gupte S. Maternal nutrition and birth size among urban affluent and rural women in India. J Am Coll Nutr. 2008;27(1):137-145.

35. Loy SL, Marhazlina M, Azwany YN, Hamid Jan JM. Higher intake of fruits and vegetables in pregnancy is associated with birth size. Southeast Asian J Trop Med Public Health. 2011;42(5):1214-1223.

36. United Nations Development Program. Human Development Report 2013. The Rise of the South: Human Progress in a Diverse World. New York, NY: United Nations Development Program; 2013. Available from: http://hdr.undp.org/sites/default/files/reports/14/hdr2013_en_complete. pdf. Accessed June 30, 2014.

37. Appel LJ, Moore TJ, Obarzanek E, et al; DASH Collaborative Research Group. A clinical trial of the effects of dietary patterns on blood pressure. N Engl J Med. 1997;336(16):1117-1124.

38. Ahn YM, Kim YJ, Park H, Park B, Lee H. Prenatal vitamin C status is associated with placental apoptosis in normal-term human pregnancies. Placenta. 2007;28(1):31-38.

39. Bergen NE, Jaddoe VW, Timmermans S, et al. Homocysteine and folate concentrations in early pregnancy and the risk of adverse pregnancy outcomes: the Generation R Study. BJOG. 2012;119(6):739-751.
International Journal of Women's Health

\section{Publish your work in this journal}

The International Journal of Women's Health is an international, peerreviewed open-access journal publishing original research, reports, editorials, reviews and commentaries on all aspects of women's healthcare including gynecology, obstetrics, and breast cancer. The manuscript management system is completely online and includes

\section{Dovepress}

a very quick and fair peer-review system, which is all easy to use. Visit http://www.dovepress.com/testimonials.php to read real quotes from published authors. 